\title{
New research in prostate cancer, ASCO-GU 2017
}

\author{
Kim Chi, MD'; Fred Saad, $M D^{2}$ \\ 'University of British Columbia, Vancouver, BC; ²Université de Montréal, Montreal, QC; Canada
}

Cite as: Can Urol Assoc J 2017;11 (6Suppl3):S166-8 http://dx.doi.org/10.5489/cuaj.4608

\section{Enzalutamide and abiraterone acetate}

At the American Society of Clinical Oncology 2017 Genitourinary Cancers Symposium (ASCO-GU), researchers presented a wealth of new data regarding the use of enzalutamide and abiraterone acetate $(A A)$ in men with metastatic castration-resistant prostate cancer (mCRPC).

Perhaps the most interesting was an analysis of androgen receptor (AR) mRNA quantification from circulating tumour cells (CTCs), with which the investigators sought to determine associations between full-length androgen receptor (AR-FL) status and clinical outcomes with either enzalutamide or AA. ${ }^{1}$ The subjects were 202 patients about to begin therapy with one of these agents, who consented to CTC collection. The clinical outcomes assessed were: prostatespecific antigen (PSA) response rate, PSA progression-free survival (PSA-PFS), clinical/radiographic progression-free survival (PFS), and overall survival (OS).

At baseline (prior to starting AA or enzalutamide), 48\% (97/202) of patients were AR-FL-negative, 26\% (52/202) were AR-FL-positive, less than the mean; 26\% (53/202) were AR-FL-positive, greater than or equal to the mean.

Higher AR-FL levels were found to be correlated with lower PSA50 responses (55.4 copies in non-responders vs. 6.7 copies in responders; $p<0.001$ ). In Kaplan-Meier analysis, PSA-PFS, PFS, and OS (Fig. 1) differed significantly between the groups.

The investigators concluded that AR-FL quantification could serve as a potential molecular biomarker of AA/ enzalutamide efficacy.

Sequencing of enzalutamide and $\mathrm{AA}$ were evaluated in several studies presented at ASCO-GU 2017. One such analysis was a phase 4, multicentre, single-arm, open-label study of enzalutamide therapy among 214 patients previously treated with AA plus prednisone. ${ }^{2}$ The median duration of enzalutamide treatment was 5.7 months (12 months in PSA responders and 4.6 months in non-PSA responders) and

the median time to PSA progression was 5.7 months $(95 \%$ confidence interval $[\mathrm{Cl}] 5.6,5.8)$. The median duration of radiographic PFS (primary endpoint) was 8.1 months. This study confirms previous retrospective reports, demonstrating that enzalutamide remains active in a subset of patients with mCRPC previously treated with AA.

A retrospective study compared the efficacy of the AA plus prednisone followed by enzalutamide sequence to the reverse sequence. ${ }^{3}$ While there were no significant differences between the agents in the first-line setting, the PSA50 response rates and median PFS-PSA were significantly better for second-line enzalutamide compared to second-line AA. The combined PFS-PSA for first- and second-line was longer for the AA-enzalutamide sequence than the enzalutamide-AA sequence (hazard ratio [HR] $0.56,95 \% \mathrm{Cl} 0.41-0.76$ ), suggesting that this sequence may be better, although OS was no different between the two groups and caution must be taken given the retrospective nature of the study.

Researchers presented a proposed multifactorial prognostic model for OS among chemotherapy-naïve men with mCRPC. ${ }^{4}$ The data used were from the PREVAIL randomized, controlled clinical trial comparing enzalutamide vs. placebo. Statistical analysis of this database revealed 11 predictive factors that were incorporated into the model: PSA, treatment, hemoglobin, neutrophil-lymphocyte ratio, liver metastases, time from diagnosis to randomization, lactate dehydrogenase, $\geq 10$ bone metastases, pain, albumin, and alkaline phosphatase.

In terms of safety, in the UPWARD study, 423 men with $\mathrm{mCRPC}$ and at least one risk factor for seizure were treated with enzalutamide (424 were enrolled but one died before drug administration occurred). ${ }^{5}$ Most of the patients' baseline seizure risk factors were medications lowering seizure threshold $(n=242)$, with the remaining having prior brain injury $(n=112)$ and cerebrovascular accident/transient ischemic attack history $(n=94)$. Four $(1.1 \%)$ evaluable patients had $\geq 1$ confirmed seizure within four months of enzalutamide initiation, with an additional four ( $1.1 \%$ ) patients having a first seizure after four months. These results suggest that enzalutamide 


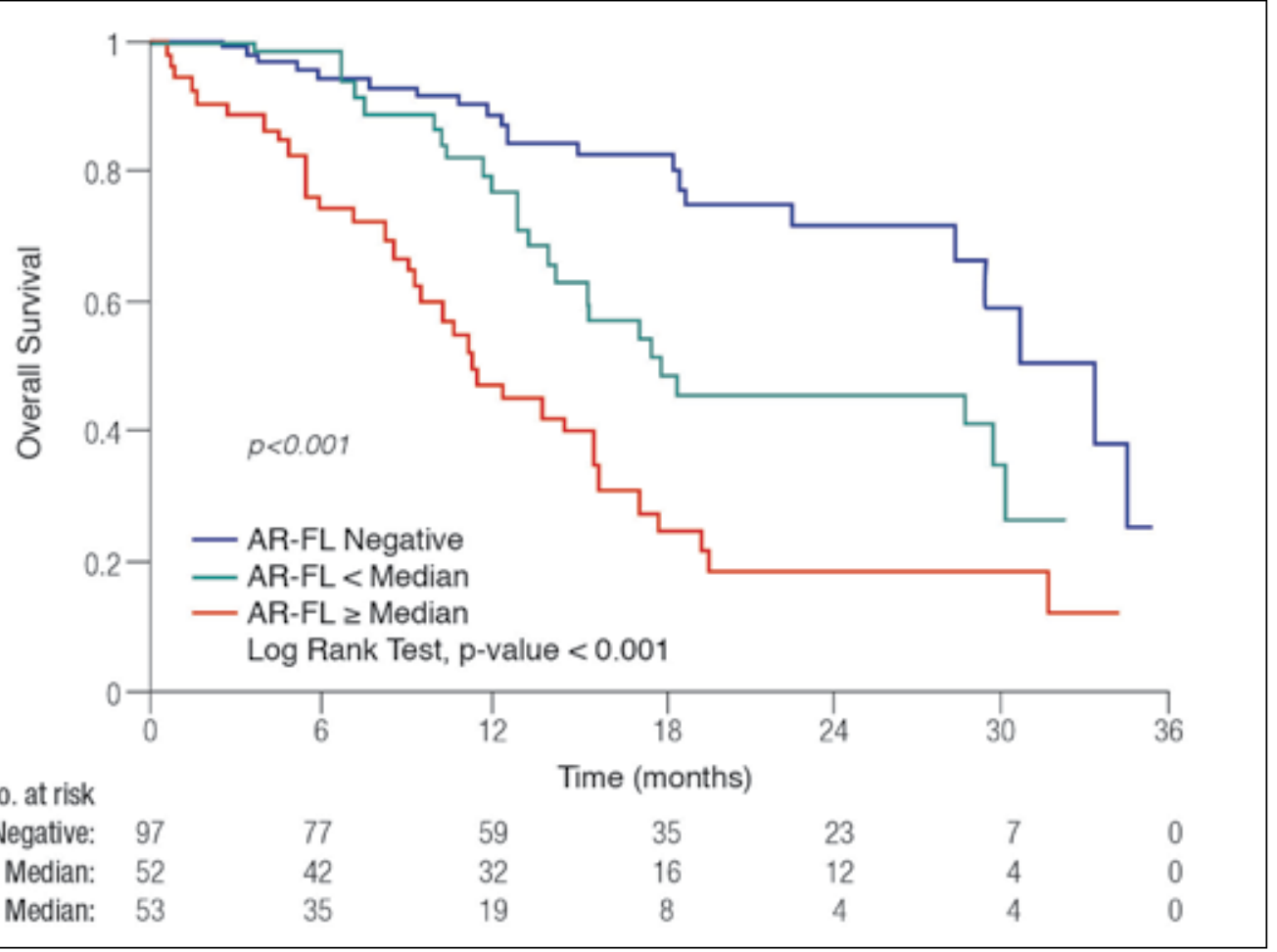

Fig. 1. Overall survival with abiraterone acetate or enzalutamide treatment by baseline full-length androgen receptor (AR-FL) status.

was not associated with a clinically significant increased risk of seizures in this higher seizure risk population.

Other researchers presented three-year followup data from a phase 2 study among 67 patients with hormone-naïve prostate cancer (HNPC) who were treated with enzalutamide. ${ }^{6}$ The key finding was that among those patients who continued to receive enzalutamide treatment at three years, more than $90 \%$ maintained a decline in PSA of $80 \%$ or more from baseline.

A separate study evaluated the efficacy and safety of combining enzalutamide with dutasteride or finasteride among 21 older (age 265 ) patients with HNPC. ${ }^{7}$ After a median 31 weeks of followup, all the patients had an ongoing PSA decline of greater than $85 \%$, without radiographic evidence of disease progression.

\section{Radium-223}

The efficacy of radium-223 therapy is being evaluated as part of an international early access program (iEAP; $n=708$ ). At ASCO-GU 2017, investigators presented the results of an analysis from this database comparing the efficacy of radium-223 among patients with or without symptoms at baseline. ${ }^{8}$ Of the 708 subjects in the iEAP, symptom status at baseline was available for 683 individuals (548 symptomatic, 135 asymptomatic).

The investigators reported that asymptomatic patients had more favourable baseline characteristics; were less likely to have received $\mathrm{AA}$, enzalutamide or docetaxel; and were more likely to receive six cycles of radium-223. Adverse events were also less frequent among patients asymptomatic at baseline. As shown in Fig. 2, OS was significantly longer among patients who were asymptomatic at baseline (HR $0.49 ; 95 \% \mathrm{Cl} 0.33-0.73)$. This study confirms the prognostic value of symptoms in patients with mCRPC.

\section{Surgery}

Researchers also presented a study investigating the utility of ${ }^{68} \mathrm{Ga}$-PSMA-positron-emission tomography/computed tomography (PET/CT) as a tool for patient selection for lymphadenectomy in addition to salvage radical prostatectomy in locally recurrent prostate cancer after primary radiotherapy. ${ }^{9}$ While the imaging was shown to have a high potential for detecting local recurrences, it had a low diagnostic accuracy for detection of lymph node metastases in local recurrences after radiotherapy.

\section{Review lectures in prostate cancer at ASCO-GU 2017}

Two of the key prostate cancer lectures given at ASCO-GU 2017 discussed the pros and cons of active surveillance (AS). ${ }^{10,11}$

Dr. Freddie Hamdy presented a review of the Prostate testing for cancer and Treatment (ProtecT) trial, the largest randomized, controlled trial to date comparing active monitoring, surgery, and radiotherapy for PSA-detected, local- 
Chi et al.

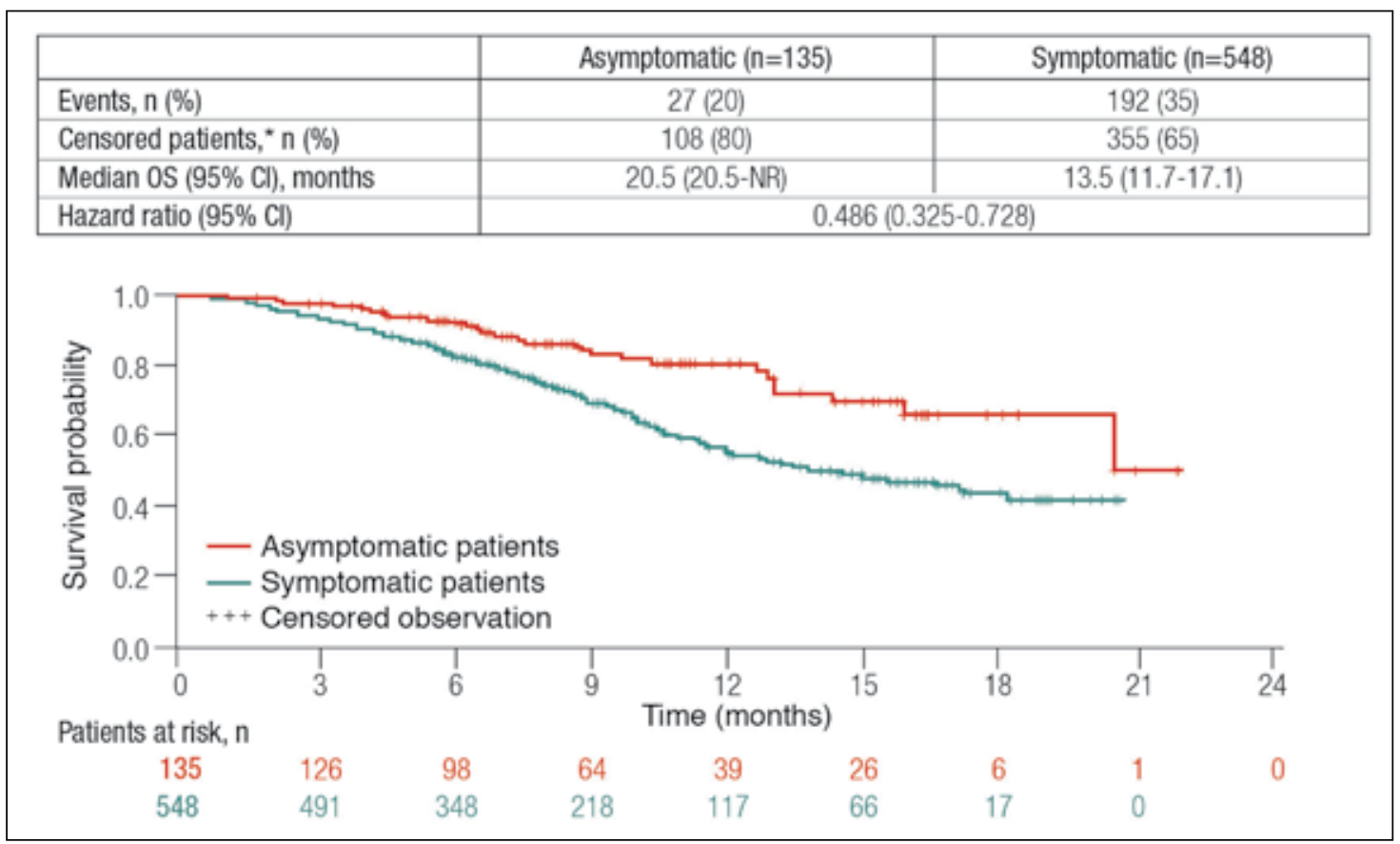

Fig. 2. Overall survival (OS) among metastatic castrate-resistant prostate cancer patients treated with radium-223 by presence or absence of symptoms at baseline. Cl: confidence interval.

ized prostate cancer (1643 randomized patients and an additional 1021 in the same three groups by patient preference).$^{10}$ Dr. Hamdy summarized the previously published findings from the study. Arguing against AS was the finding that there was a $50 \%$ reduction in metastases with radical treatments compared to AS. He also showed previously unpublished data for 17 men who died of prostate cancer in the study. Of these, $13 / 17$ had AS as their first treatment; however, $44 \%$ of men on active monitoring avoided treatment altogether, and approximately $80 \%$ of men had no sign of progression. Patient-reported outcomes (e.g., erectile firmness, incontinence) were least favourable among those patients who underwent prostatectomy.

Dr. Shonit Punwani discussed the role of imaging modalities to guide AS. ${ }^{11}$ At enrolment, he explained that imaging (e.g., multiparametric magnetic resonance imaging) can help tell us whether the tumour has been adequately sampled at baseline and whether there is any disease outside the prostate; however, its use to evaluate and capture progression is problematic, as progression is not clearly defined.

\section{References}

1. Silberstein J, Luber B, Wang H, et al. Clinical significance of AR mRNA quantification from circulating tumour cells (CTCs) in men with metastatic castration-resistant prostate cancer (mCRPC) treated with abiraterone (Abi) or enzalutamide (Enza). Presented at ASCO-GU 2017; abstract \#132.

2. De Bono IS, Chowdhury S, Feyerabend S, et al. Efficacy and safety of enzalutamide (ENZA) in patients with metastatic castration-resistant prostate cancer $(\mathrm{m}(\mathrm{RPC})$ previously treated with abiraterone acetate (Abi): A multicentre, single-arm, open-label study. Presented at ASCO-GU 2017; abstract \#165.

3. Terada N, Maughan BL, Akamatsu S, et al. Exploring optimal sequence of abiraterone and enzalutamide in patients with castration-resistant prostate cancer: The Kyoto-Baltimore collaboration. Presented at ASCO-GU 2017; abstract \#219.

4. Armstrong AJ, Lin P, Higano CS, et al. Development and validation of a prognostic model for overall survival in chemotherapy-naive men with metastatic castration-resistant prostate cancer $(\mathrm{mCRPC})$ from the phase 3 PREVAll clinical trial. Presented at ASCO-GU 2017; abstract \#138.

5. Slovin SF, Clark W, Carles J, et al. Seizure rates in enzalutamide (ENZ)-treated men with metastatic castration-resistant prostate cancer (mCRPC) at increased risk of seizure: UPWARD study. Presented at ASCO-GU 2017; abstract \#147.

6. Tombal BF, Borre M, Rathenberg $\mathrm{P}$, et al. Long-term efficacy and safety of enzalutamide (ENZ) monotherapy in hormone-naïve prostate cancer (HNPC): Three-year, open-label, followup results. Presented at ASCO-GU 2017; abstract \#220.

7. Kilari D, Guancial E, Sahasrabudhe D, et al. A phase 2 study of enzalutamide (Enz) with dutasteride (Dut) or finasteride (Fin) in men $\geq 65$ years with hormone-naive systemic prostate cancer (HNSPCa). Presented at ASCO-GU 2017; abstract \#179.

8. Heidenreich A, Gillessen S, Heinrich D, et al. Radium-223 (Ra-223) in asymptomatic metastatic castrationresistant prostate cancer (mCRPC) patients treated in an international early access program (iEAP). Presented at ASCO-GU 2017; abstract \#158.

9. Pfister D, Heidegger I, Verburg F, et al. Accuracy of ${ }^{68} \mathrm{Ga}-\mathrm{PSMA}-\mathrm{PET} / \mathrm{CT}$ for patient selection for lymphadenectomy in addition to salvage radical prostatectomy in locally recurrent prostate cancer after primary radiotherapy. Presented at ASCO-GU 2017; abstract \#229.

10. Hamdy F. What Protect tells us about active surveillance? Presented at ASCO-GU 2017.

11. Punwani S. Imaging to guide active surveillance. Presented at ASCO-GU 2017. 